\title{
PHYSICAL CHARACTERISTICS OF V 1016 CYGNI
}

\author{
F. CIATTI, A. MAMMANO, and L. ROSINO \\ Asiago Artrophysical Observatory, Italy
}

\begin{abstract}
Short description of the spectral phenomena of V 1016 Cyg.
\end{abstract}
The peculiar variable star V 1016 Cygni ( $\mathrm{MH} \alpha 328-116)$ has been observed at Asiago since the announcement of its brightening. During 1963-1971 its magnitude rose from 15.5 to 10.8. More recent observations made at Asiago on photographic material taken with the Schmidt telescopes give the following mean values: $\langle B\rangle=10.55 \pm 0.15$, $\langle V\rangle=10.65 \pm 0.15$. Small brightness fluctuations may be present.

The 1965-1971 spectrum showed many strong emission lines, most of which were forbidden lines of higher and higher ionization potential (Ciatti et al. 1971). In the period 1971-1974 the emission spectrum $(\lambda \lambda 3700-10900)$ indicates a further increase of the excitation, as inferred from the strengthening lines of the following ions: He II $(\lambda \lambda 4199,4541,10123),[\mathrm{Ar} \mathrm{v}](\lambda \lambda 6435,7006),[\mathrm{Ca} \mathrm{v}](\lambda \lambda 5308,6085),[\mathrm{Fe} \mathrm{vI}]$ $(\lambda \lambda 5335,5425)$, and [Fe vII] $(\lambda \lambda 4893,4944,5159,5721,6085)$. The [Fe VII] line at $6085 \AA$, which was of moderate intensity in 1971 , is now outstanding.

Moreover, the presence of absorption features due to TiO $(\lambda \lambda 7600,8200,8430)$ and VO $(\lambda \lambda 7900,10460)$ is observed in the near infrared region. These molecular bands are indicative of a cool source of spectral type about M6, which is in agreement with the red continuum of V $1016 \mathrm{Cyg}$ (see also Boyarchuk, 1968). They can be ascribed to the cool variable star observed before 1963 and recently by Harvey (1974) in the infrared.

V 1016 Cyg is thus characterized by a combination spectrum showing a very hot source of radiation and features of a cool component. This latter, which is thought to be responsible for the strong infrared excess (Allen, 1973), is very likely loosing mass, thus producing the large gaseous envelope which is excited by the hot source, as shown by forbidden lines and radio emission. Both optical and radio observations indicate a steady flow with low expansion velocity (FitzGerald, 1973; Seaquist and Gregory, 1973).

In spite of the presence of a combination spectrum, the peculiar object V $1016 \mathrm{Cyg}$ differs from the classical symbiotic stars. From some points of view, its morphological characteristics allow a comparison with other classes of objects, like very slow novae, compact planetary nebulae, and BQ[ ] radio-stars with infrared excess (see Ciatti and Mammano, this Symposium, p. 409).

The brightening of V 1016 Cyg from $15^{m} .5$ to about $10^{m} .8$ and the presence of a very strong emission spectrum with permitted and forbidden lines of increasing excitation are probably to be ascribed to an increase of temperature of the hot star, and consequently to the conversion of ultraviolet into visual radiation.

We further suggest that continuous ejection of matter from the cool component of 
V 1016 Cyg will finally produce a compact planetary nebula, of which we are now observing an early stage.

The full paper will be submitted to Astronomy and Astrophysics.

\section{References}

Allen, D. A.: 1973, Monthly Notices Roy. Astron. Soc. 161, 145.

Boyarchuk, A. A.: 1968, Astrofizika 4, 289.

Ciatti, F., Mammano, A., and Rosino, L.: 1971, Veröff. Remeis Sternwarte Bamberg (Colloq. on Variable Stars) 9, 64.

FitzGerald, M. P.: 1973, Nature 245, 58.

Harvey, P. M.: 1974, Astrophys. J. 188, 95.

Seaquist, E. R. and Gregory, P. C.: 1973, Nature 245, 85. 